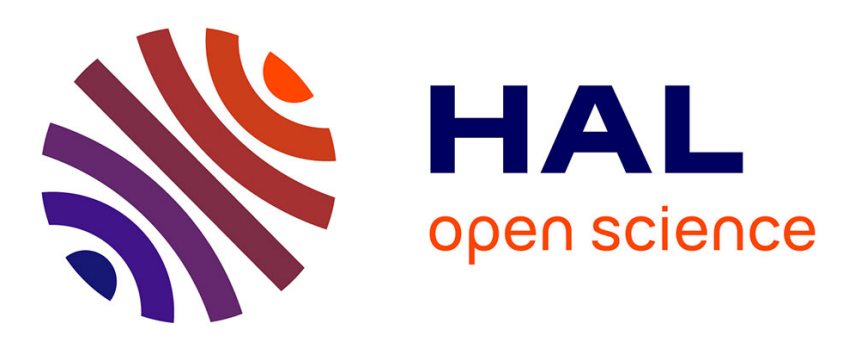

\title{
Etude par diffraction X d'un acier inoxydable traité par implantation ionique à haute énergie
}

\author{
V. Lallemand, D. Muller, P. Mille, A. Cornet, P. Denier, J. Stoquert, J. Grob
}

\section{To cite this version:}

V. Lallemand, D. Muller, P. Mille, A. Cornet, P. Denier, et al.. Etude par diffraction X d'un acier inoxydable traité par implantation ionique à haute énergie. Journal de Physique IV Proceedings, 1996, 06 (C4), pp.C4-475-C4-480. 10.1051/jp4:1996444 . jpa-00254327

\section{HAL Id: jpa-00254327 https://hal.science/jpa-00254327}

Submitted on 1 Jan 1996

HAL is a multi-disciplinary open access archive for the deposit and dissemination of scientific research documents, whether they are published or not. The documents may come from teaching and research institutions in France or abroad, or from public or private research centers.
L'archive ouverte pluridisciplinaire HAL, est destinée au dépôt et à la diffusion de documents scientifiques de niveau recherche, publiés ou non, émanant des établissements d'enseignement et de recherche français ou étrangers, des laboratoires publics ou privés. 


\title{
Etude par diffraction $X$ d'un acier inoxydable traité par implantation ionique à haute énergie
}

\author{
V. Lallemand, D. Muller*, P. Mille, A. Cornet, P. Denier, J.P. Stoquert* et J.J. Grob* \\ ENSAIS, LMCM, 24 boulevard de la Victoire, 67084 Strasbourg cedex, France \\ * CNRS, Laboratoire PHASE, BP. 20, 67037 Strasbourg cedex 2, France
}

Résumé: Une forte dose d'azote a été implantée à l'énergie de $1 \mathrm{MeV}$ dans un acier austénistique pour en modifier les propriétés mécaniques et le rendre apte à des applications biomédicales. La rétrodiffusion Rutherford a montré qu'il est nécessaire d'éviter l'échauffement de l'échantillon pendant l'implantation, l'azote diffusant rapidement dans l'acier. La diffraction de rayons $\mathrm{X}$ a permis de mettre en évidence et de suivre les phases qui se succèdent au cours des recuits à des températures croissantes.

\begin{abstract}
MeV}$ high dose nitrogen implantation was used to modify the mechanical properties of an austenistic steel. Rutherford backscattering analysis showed that it is necessary to implant at low temperature to avoid nitrogen diffusion. Phase identification and other structural informations were obtained by $\mathrm{X}$-rays diffraction, as a function of implant dose and annealing temperature.
\end{abstract}

\section{INTRODUCTION.}

Certains aciers inoxydables sont utilisés pour réaliser des implants chirurgicaux ou des pièces d'ostéosynthèse. Pourtant, ces matériaux n'ont pas des caractéristiques mécaniques ou de biocompatibilité comparables à celles du titane pur ou allié. De nombreux travaux ont montré qu'un traitement superficiel par un faisceau d'ions azote améliore considérablement les propriétés tribologiques (friction, résistance à l'usure) de la plupart des métaux et de leurs alliages [1-4]. A forte dose, ce traitement peut aussi conduire à la formation d'une barrière qui stoppe la diffusion des éléments métalliques, évitant leur dissolution dans l'environnement physiologique [5]. La nitruration des aciers par implantation d'azote est un phénomène complexe et encore mal connu qui dépend, bien sûr, de la composition de l'acier, mais aussi des paramètres du traitement: énergie des ions, dose, température de la cible pendant l'implantation ou, éventuellement, pendant le recuit thermique [6,7]. Dans la plupart des cas, les ions sont implantés à basse énergie (quelques dizaines de $\mathrm{keV}$ ), ce qui conduit à une nitruration sur quelques milliers d'angstroms $[8,9]$. L'utilisation d'ions de haute énergie permet de traiter des épaisseurs plus grandes, typiquement sur 1 à $5 \mu \mathrm{m}$ [10]. Le but de cette étude préliminaire est donc d'observer l'éventuelle apparition de nitrures lors d'implantations haute énergie dans un acier d'intérêt biologique en fonction de la température pendant l'implantation ou pendant le recuit. 


\section{PREPARATION DES EChaNTILlONS ET TEChNiQUeS D'ANALYSE.}

\subsection{Les échantillons.}

Le matériau utilisé dans cette étude est l'acier austénitique Z2CND17-12 de composition atomique nominale: $\mathrm{Fe} 70 \%, \mathrm{Cr} 17 \%$, Ni $12 \%$ et Mo $1 \%$. Les échantillons, des disques de 2 $\mathrm{cm}$ de diamètre et de $3 \mathrm{~mm}$ d'épaisseur, ont subi différents types de polissage, mécanique ou électrochimique. Les meilleures surfaces, du point de vue de la rugosité, ont été obtenues par polissage mécanique à l'aide d'une pâte au diamant jusqu'à une taille de grain de 1 micron.

\subsection{L'implantation ionique.}

Les échantillons ont ensuite été placés sur différents supports suivant la température d'implantation souhaitée: isolés thermiquement, suivant l'énergie apportée par le faisceau d'ions (c'est à dire le produit courant-tension), ils pouvaient s'échauffer jusqu'à des températures comprises entre 300 et $700^{\circ} \mathrm{C}$; fixés à la pâte d'argent sur un support relié à un réservoir contenant de l'azote liquide, leur température restait inférieure à $20^{\circ} \mathrm{C}$ quel que soit le courant d'implantation. Dans la suite de ce travail, ces deux façons d'implanter seront respectivement désignées par les termes "haute" ou "basse" température. Le vide résiduel dans l'enceinte contenant les cibles était de l'ordre de $5 \times 10^{-7}$ Torr. Pour obtenir des profondeurs de pénétration de l'ordre du micromètre, une énergie de $1 \mathrm{MeV}$ a été utilisée. Plus précisemment, le faisceau, constitué de la molécule $\mathrm{N}_{2}$ une fois chargée, était accéléré sous une tension de $2 \mathrm{MV}$. La dose d'ions ainsi implantés a été variée entre $10^{17}$ et $10^{18}$ ions $/ \mathrm{cm}^{2}$.

Après implantation, une partie des plaquettes a subi un traitement thermique de recuit pendant 5 heures dans un four sous flux de gaz inerte (argon), à des températures croissantes de 280,410 et $510^{\circ} \mathrm{C}$.

\subsection{Analyses des échantillons.}

Le profil de l'azote implanté dans cet acier a été déterminé par rétrodiffusion de particules légères (RBS). Le faisceau d'analyse, issu de l'accélérateur Van de Graaff $4 \mathrm{MV}$ du Centre de Recherches Nucléaires de Strasbourg, était constitué d'ions $\mathrm{He}^{+}$de $3 \mathrm{MeV}$. L'énergie des particules rétrodiffusées dans un angle de 160 degrés était mesurée a l'aide d'un détecteur à barrière de surface, l'acquisition et le traitement des données étant réalisés au moyen des logiciels TMCA et RUMP [11].

L'identification des phases et les autres modifications de structure des échantillons ont été étudiées par diffraction X à l'aide d'un diffractomètre SIEMENS D500 dans une géométrie standard $\theta / 2 \theta$ et utilisant, comme source, la raie $K \alpha$ du Cobalt. Tous les échantillons ont été analysés en utilisant les mêmes conditions expérimentales.

\section{RESULTATS ET DISCUSSION.}

\subsection{Profil des ions implantés.}

Le spectre RBS de la figure 1 correspond à un échantillon implanté à la dose la plus élevée (1018 ions $/ \mathrm{cm}^{2}$ ), à "basse" température (de l'ordre ou inférieure à l'ambiante) et n'ayant subi aucun traitement thermique. La distribution en énergie des particules rétrodiffusées sur l'acier présente une chute du rendement caractéristique de la présence de l'azote en profondeur. La ligne continue est le résultat d'une simulation où l'on a supposé que l'azote est réparti selon une distribution gaussienne à la profondeur moyenne de $0.73 \mu \mathrm{m}$, avec une largeur à mihauteur de $0.28 \mu \mathrm{m}$. Ces paramètres sont très voisins des valeurs mesurées par d'autres auteurs [10] et de ceux du profil théorique calculés à l'aide des simulations TRIM [12] et PROFILE [13] . La comparaison de la figure 2 montre que la distribution mesurée, extraite du spectre RBS, est un peu plus large que le profil calculé. La différence pourrait être due à une légère diffusion de l'azote. Toutefois, la dose utilisée pour ajuster le spectre RBS expérimental 
correspond exactement à la quantité d'ions effectivement implantée. En d'autres termes, il n'y a pas d'exodiffusion. Ces résultats montrent que l'azote, à cette température, ne s'étale pas de façon significative dans l'acier sous l'effet de la diffusion. Ce n'est pas le cas lors d'implantations à "hautes" températures (supérieures à $300^{\circ} \mathrm{C}$ ), où nous constatons une forte diffusion, en accord avec les résultats de Jagielski et col. [14] ou de Vredenberg et col. [10]. On remarquera aussi qu'à cette dose et en l'absence de diffusion, l'azote atteint, au maximum de la distribution, une concentration de l'ordre de $30 \%$ atomique.

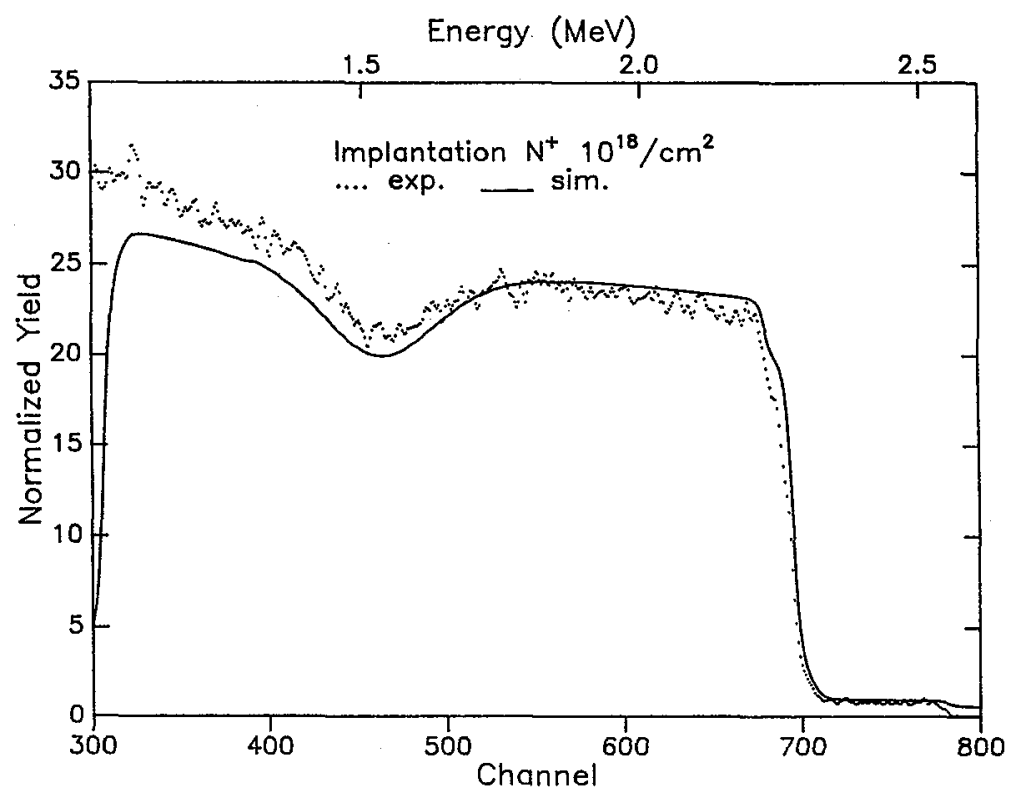

Fig.1 Spectre RBS de l'acier Z2CND17-12 implanté d'ions azote de $1 \mathrm{MeV}$ et à la dose de $10^{18}$ ions/ $\mathrm{cm}^{2}$. La ligne continue correspond à la simulation RUMP [11] du spectre expérimental.

\subsection{Phases formées au cours de l'implantation et du recuit.}

La diffraction $\mathrm{X}$ a donc été utilisée pour suivre la séquence des phases de nitrure formées, en fonction de la dose et du traitement de recuit. Notons d'abord que les implantations à haute température n'ont pas permis de révéler la trace d'un quelconque nitrure, ce qui est probablement dû à la diffusion et la dissolution de l'azote vers la profondeur de l'échantillon et/ou, éventuellement, à l'exodiffusion. La figure 3 montre deux diffractogrammes correspondant à l'alliage avant et après l'implantation basse température à la dose de $10^{18}$ ions $/ \mathrm{cm}^{2}$. On y détecte la prédominance des pics de l'austénite, et, après implantation, l'apparition, à la base de chaque pic d'austénite, d'une distribution secondaire que l'on a décomposé en une somme de plusieurs contributions à l'aide du logiciel DIFFRACT (encart sur la figure 3). Cette décomposition révèle l'apparition de deux pics, l'un, bien résolu, pourrait correspondre à la présence de nitrure de nickel et surtout de fer et l'autre, très large, noté $\gamma_{\mathrm{N}}$, est attribué à la présence d'azote en solution dans l'austénite et qui induirait des contraintes dans l'arrangement cfc du fer . 


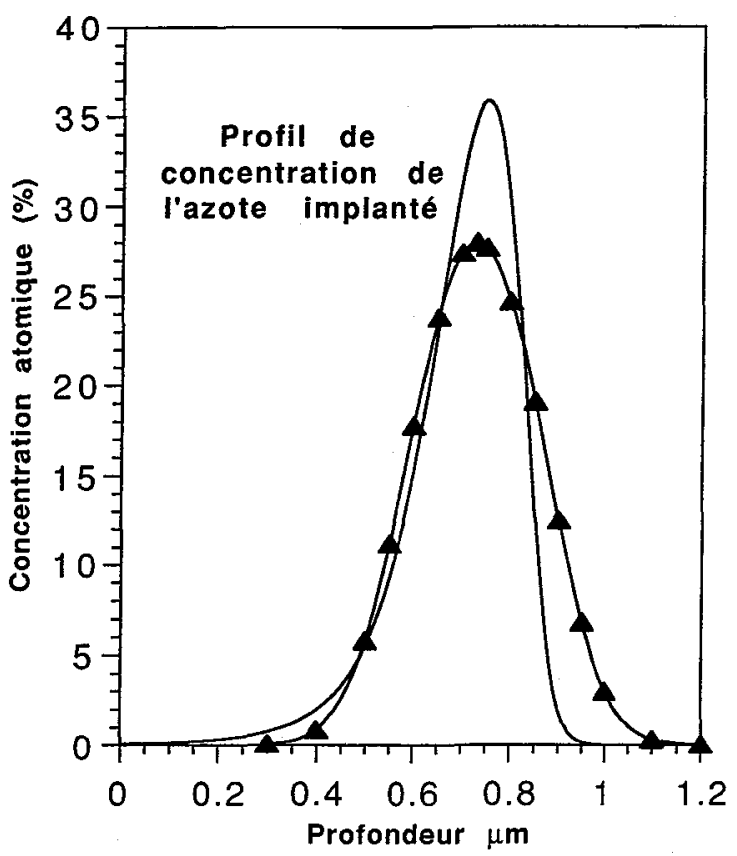

Fig.2 Profil de concentration de l'azote implanté déterminé à l'aide du logiciel PROFILE [13]. Le profil expérimental est déduit du spectre RBS de la figure 1.

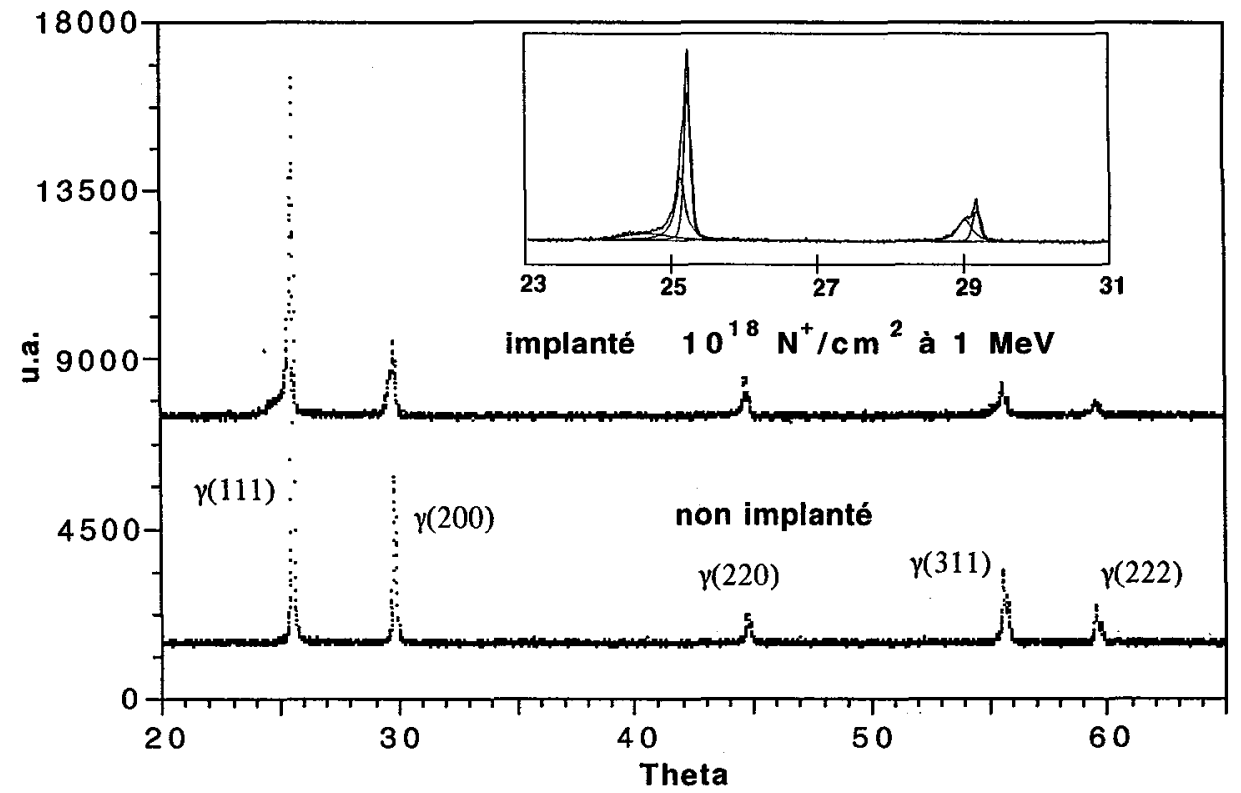

Fig. 3 Diffractogrammes RX enregistrés en mode $\theta / 2 \theta$ sur un échantillon d'acier avant et après l'implantation d'une dose de $10^{18} \mathrm{~N}^{+} / \mathrm{cm}^{2}$ à $1 \mathrm{MeV}$. L'encart représente une déconvolution des deux principaux pics de l'échantillon implanté. 


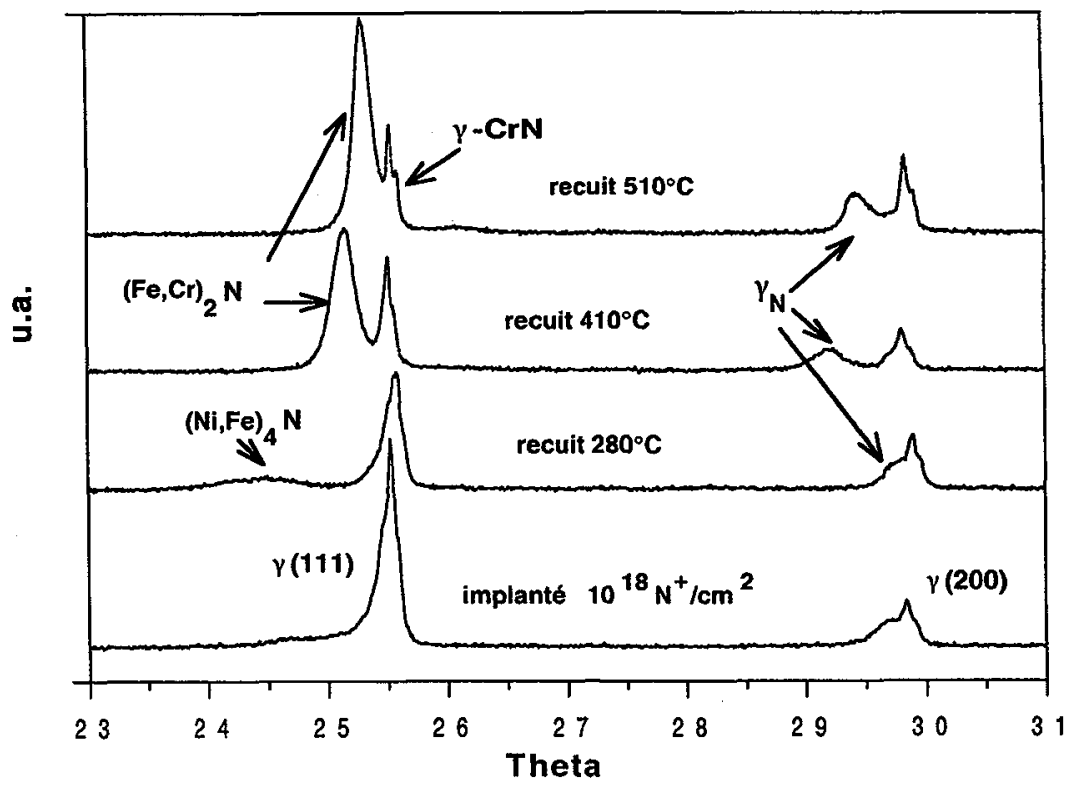

Fig. 4 Diffractogrammes RX enregistrés en mode $\theta / 2 \theta$ sur un échantillon implanté à la dose de $10^{18} \mathrm{~N}^{+} / \mathrm{cm}^{2}$ à $1 \mathrm{MeV}$ suivi de recuits pendant 5 heures à différentes températures.

La figure 4 montre quatre diffractogrammes correspondant à l'échantillon implanté à la plus forte dose et à basse température, respectivement non recuit, recuit à 280 , à 410 et à $510^{\circ} \mathrm{C}$. Il est à noter qu'une analyse quantitative détaillée en fonction de la température du recuit semble délicate dans la mesure où, comme l'ont démontré plusieurs travaux [9,14], l'azote peut à nouveau diffuser dans le fer ou l'acier à des température supérieures à $350^{\circ} \mathrm{C}$. Nous nous contenterons donc ici d'une description qualitative des phases présentes. Le spectre de l'échantillon recuit à $280^{\circ} \mathrm{C}$ fait clairement apparaître la présence des nitrures $\mathrm{Fe}_{4} \mathrm{~N}$ et $\mathrm{Ni}_{4} \mathrm{~N}$. Les pics attribués précédemment à l'azote en solution dans l'austénite ont presque disparu. A $410^{\circ} \mathrm{C}$, on constate d'autres transformations: les nouveaux nitrures formés sont $\mathrm{Fe}_{2} \mathrm{~N}$ et $\mathrm{Cr}_{2} \mathrm{~N}$; les nitrures présents à $280^{\circ} \mathrm{C}$ ont disparu; l'austénite est en forte diminution et les pics caractéristiques de la présence de l'azote en solution réapparaissent. Le spectre obtenu sur une plaquette recuite à $510^{\circ} \mathrm{C}$ est assez voisin du précédent. Cependant, la phase $\mathrm{CrN}$ apparait. La quantité de $\mathrm{Cr}_{2} \mathrm{~N}$ et de $\mathrm{Fe}_{2} \mathrm{~N}$ est en augmentation alors que l'austénite continue de diminuer. Le tableau 1 résume l'ensemble de ces observations.

Tableau 1. Résumé de l'ensemble des observations par diffraction $X$ sur des échantillons implantés à différentes doses ou recuits à différentes températures.

\begin{tabular}{|c|c|c|}
\hline Dose (ions/cm & Température de recuit \\
$\left({ }^{\circ} \mathrm{C}\right)$ & Phases présentes \\
\hline non implanté & - & fer $\gamma$ \\
\hline $4 \times 10^{17}$ & - & $\mathrm{Ni}_{4} \mathrm{~N}, \mathrm{Ni} \mathrm{N}_{3} \mathrm{~N}$ \\
\hline $10^{18}$ & - & $\mathrm{Ni}_{4} \mathrm{~N}, \mathrm{Ni}_{3} \mathrm{~N}, \mathrm{Fe}_{3} \mathrm{~N}, \mathrm{~N}_{\mathrm{N}}$ \\
\hline $10^{18}$ & 280 & $\mathrm{Ni}_{4} \mathrm{~N}, \mathrm{Fe}_{4} \mathrm{~N}$ \\
\hline $10^{18}$ & 410 & $\mathrm{Fe}_{2} \mathrm{~N}, \mathrm{Cr}_{2} \mathrm{~N}, \mathrm{~N}$ \\
\hline $10^{18}$ & 510 & $\mathrm{Fe}_{2} \mathrm{~N}, \mathrm{Cr}{ }_{2} \mathrm{~N}, \mathrm{CrN}, \mathrm{N}_{\mathrm{N}}$ \\
\hline
\end{tabular}


La séquence d'apparition des nitrures, nitrures de nickel à basse température et de chrome à plus haute température a déjà été observée par Williamson et col. [6].

\section{CONCLUSION.}

Cette étude a montré que l'implantation d'azote à haute énergie, à forte dose et à basse température dans un acier austénistique, suivie d'un traitement thermique de recuit à une température supérieure à $400^{\circ} \mathrm{C}$, semble progressivement provoquer la décomposition de l'austénite au profit de la formation de nitrures de fer et de chrome. L'azote excédentaire forme une solution solide dans l'austénite, qui, d'après certains auteurs, constitue une couche dure et résistante [6].

\section{REFERENCES:}

[1] G. Dearnaley, Nucl. Instr. and Methods B24/25 (1987) 506.

[2] F. Cerny, M. Prazak and J.Suchanek, Nucl. Instr. and Methods B59/60 (1991) 762.

[3] R. Wei, P.J. Wilbur, O. Ozturk and D.J. Williamson, Nucl. Instr. and Methods, B59/60 (1991) 731.

[4] M Guemmaz, A Mosser, D. Raiser, J.J. Grob, A. Cornet and S. Paletto, Proceedings of the MRS fall meeting, Boston (USA), 28 nov.-2 dec. 1994.

[5] T. Rostund, P. Thomsen, L.M. Biursten and L.E. Ericson, J. Biomed. Mat. Res. 24 (1990)847.

[6] D.L. Williamson, O. Ozturk, S. Glick, R. Wei and P.J. Wilbur, Nucl. Instr. and Methods B59/60 (1991) 737.

[7] R. Wei, P.J. Wilbur, O. Ozturk and D.L. Williamson, Nucl. Instr. and Methods B59/60 (1991) 731.

[8] A. Chayahara, S. Nakashima, M. Hashimoto, T. Sasaki, M. Kurokawa, S. Kiyama and M. Satou Nucl. Instr. and Methods B59/60 (1991)893.

[9] M. A. El Khakani, G. Marest, N. Moncoffre and J. Tousset, Nucl. Instr. and Methods B59/60 (1991)817.

[10] A.M. Vredenberg, C.M. Perez-Martin, J.S. Custer, D.O. Boerma, L. de Wit, F.W. Saris, N.M. v/d Pers, Th. H. de Keijser and E.J. Mittemeijer Surface Coatings Technol. 51 (1992)79.

[11] L.R. Doolittle, Nucl. Instr. and Methods, B9 (1985) 334.

[12] J.F. Ziegler and J.P. Biersack, "The stopping. and range of ions in solids", Pergamon Press, New-York (1985), ISBN-0-08-021603-X.

[13] S.N. Bunker and A.J. Armini, Nucl. Instr. and Methods, B39 (1989) 7.

[14] J. Jagielski, G. Gawlik, A. Podgorski, A. Turos, A. Dygo and N. Madi, Phys. Stat. Sol. (a), 112 (1989) 343. 